Date last updated: October 15, 2012. Date last accessed: August 2, 2012.

4 Johnston SD, Kempston A, Robinson TJ. Pneumonitis secondary to the influenza vaccine. Postgrad Med J 1998; 74: 541-542.

5 Heinrichs D, Sennekamp J, Kirsten A, et al. Allergische Alveolitis nach Grippeschutzimpfung [Allergic alveolitis after influenza vaccination]. Pneumologie 2009; 63: 508-511.

6 Kanemitsu Y, Kita H, Fuseya Y, et al. [Interstitial pneumonitis caused by seasonal influenza vaccine]. Nihon Kokyuki Gakkai Zasshi 2010; 48: 739-742.

7 Bhurayanontachai R. Possible life-threatening adverse reaction to monovalent H1N1 vaccine. Crit Care 2010; 14: 422.
8 Umeda Y, Morikawa M, Anzai M, et al. Acute exacerbation of idiopathic pulmonary fibrosis after pandemic influenza A (H1N1) vaccination. Intern Med 2010; 49: 2333-2336.

9 Kumamoto T, Mitsuyama H, Hamasaki T. [Case report; drug induced lung injury caused by 2009 pandemic H1N1 vaccine]. Nihon Naika Gakkai Zasshi 2011; 100: 3034-3037.

10 Matsuno O. Drug-induced interstitial lung disease: mechanisms and best diagnostic approaches. Respir Res 2012; 13: 39.

11 Pichler WJ, Tilch J. The lymphocyte transformation test in the diagnosis of drug hypersensitivity. Allergy 2004; 59: 809-820.

DOI: $10.1183 / 09031936.00146912$

\title{
Bronchial endoscopic ultrasound elastography: preliminary feasibility data
}

\section{To the Editor:}

Medical elastography consists of biomechanically characterising a zone of tissue on the basis of its response to the application of mechanical stress. This stress can be quasistatic (local compression) or vibratory (propagation of shear waves). In the various medical applications of elastography, the response to the stress is described by mapping the tensile modulus, or Young's modulus. Young's modulus corresponds to the slope of the stress-strain relationship measured during a series of tensile tests [1]. The elasticity of a tissue depends on its nature, its state (fat infiltration or fibrosis) and its homogeneity. A tumour situated in a zone of healthy tissue can, therefore, be detected by its decreased elasticity. The tumour can also be described in space, based on the principle that within anisotropic materials (typically represented by heterogeneous tissues), the value of Young's modulus varies as a function of the direction of the force applied to the material tested. Simple colour coding of the tensile response provides mapping of the elasticity of the zone examined. Very hard tissues are generally coded as blue, while soft tissues are coded as red and intermediate tissues are coded as green.

Elastography is now used in various fields of medicine, often in combination with ultrasound (ultrasound elastography, sometimes called computer-assisted palpation). It has been applied to the diagnosis of breast [2], thyroid [3] and prostate tumours [4], in vascular disease [5], and in hepatology [6]. Elastography has also been combined with gastrointestinal endoscopic ultrasound to investigate pancreatic masses [7] and nodal invasion by rectal cancer [8]. A recent meta-analysis of the performances of gastrointestinal endoscopic ultrasound elastography to distinguish between benign and malignant lymph nodes concluded to a sensitivity of $88 \%$, a specificity of $85 \%$ and an area under the receiver operating characteristics curve of 0.9456 [9]. These performances are superior to those of endoscopic ultrasound alone [10].

To our knowledge, elastography in combination with bronchial endoscopic ultrasound has not yet been evaluated. We report preliminary feasibility data and the first evaluation of this technique. This study was approved by the local ethics committee (Comité de Protection des Personnes Ile-de-France 6 Pitié-Salpêtrière, Paris, France).

All patients referred to our centre for assessment of mediastinal lymphadenopathy between February and May 2012 were studied by bronchial endoscopic ultrasound elastography under light general anaesthesia (10 patients, 13 lymph node areas measuring 10-30 mm). Real-time elastographic mapping was performed using an ultrasound elastography module incorporated into a ultrasound machine (Hi-vision Avius $\mathbb{B}$; Hitachi Medical Systems, Kashiwa, Japan) coupled with a bronchial endoscopic ultrasound probe (EB1970 video bronchoscope; Pentax, Tokyo, Japan). Elasticity colour mapping was performed for each lymph node studied by superimposing the colour coding of tensile responses with the endoscopic B-mode ultrasound image and by defining the frequency histogram of the responses in the zone studied. Transbronchial needle aspiration (TBNA) was performed in each case using a 22-gauge needle (sono Tip ${ }^{\circledR}$ EBUS; MediGlobe, Rosenheim, Germany) by targeting, as far as possible, the zone identified as being the least elastic.

Colour mapping of the tissue studied and the corresponding elasticity histogram were obtained in every case (fig. 1). The elastography module proved easy to use and prolonged the examination time by only a few minutes. The five lymph nodes demonstrated to be malignant on histological examination of the TBNA material were characterised by decreased elasticity (dominant blue colour, elasticity ranging from 10 to 49 on the histogram and $>80 \%$ of the tissue considered to be "hard" in the target zone) (table 1). No malignant cell was identified in the other eight lymph nodes (elasticity ranging from 55 to 167 and $6-71 \%$ hard zones) regardless of the final diagnosis. Although discussing specificity and sensitivity is not reasonably possible with such a small sample, these preliminary results are consistent with the results published for gastrointestinal endoscopic ultrasound $[9,10]$. Notably, mediastinoscopy was not performed in those of our patients where TBNA did not provide diagnostic proof because a therapeutic decision was taken based on other factors (table 1). In future 

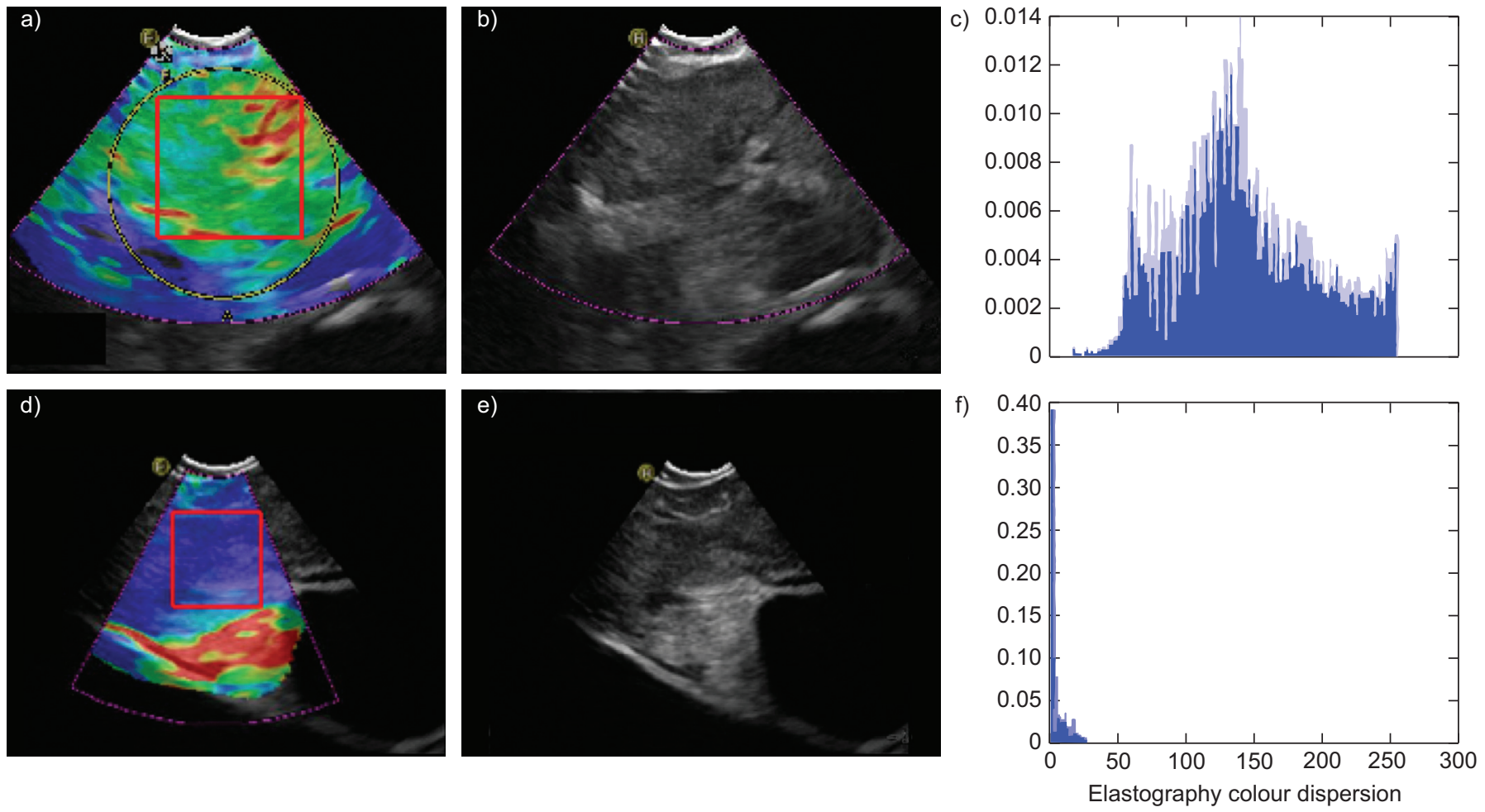

FIGURE 1. Screenshots from the elastography module linked to an endoscopic ultrasound. The histograms show the elastography colour dispersion in the region of interest defined and also give the mean of elasticity ( 0 : coded in blue; 255: coded in red). a-c) A benign lymph node is compressible (coded as green (a) and histogram centred around 150 (c)) whereas d-e) a malignant lymph node appears less compressible (coded as blue (d) and histogram is around 20 with a low colourimetric mean (f)). b) and e) correspond to the ultrasound images on top of which the elastographic signal is superimposed ( $a$ and d, respectively). c, f) The $x$-axis displays the range of relative strain values in the measurement region of interest (ROI), assigned to an 8-bit colour scale (0-255); the $y$-axis represents the frequency of occurrence of the various strain values (that is, the number of pixels in the ROI that have that particular strain value).

TABLE 1 Elastographic and histological data

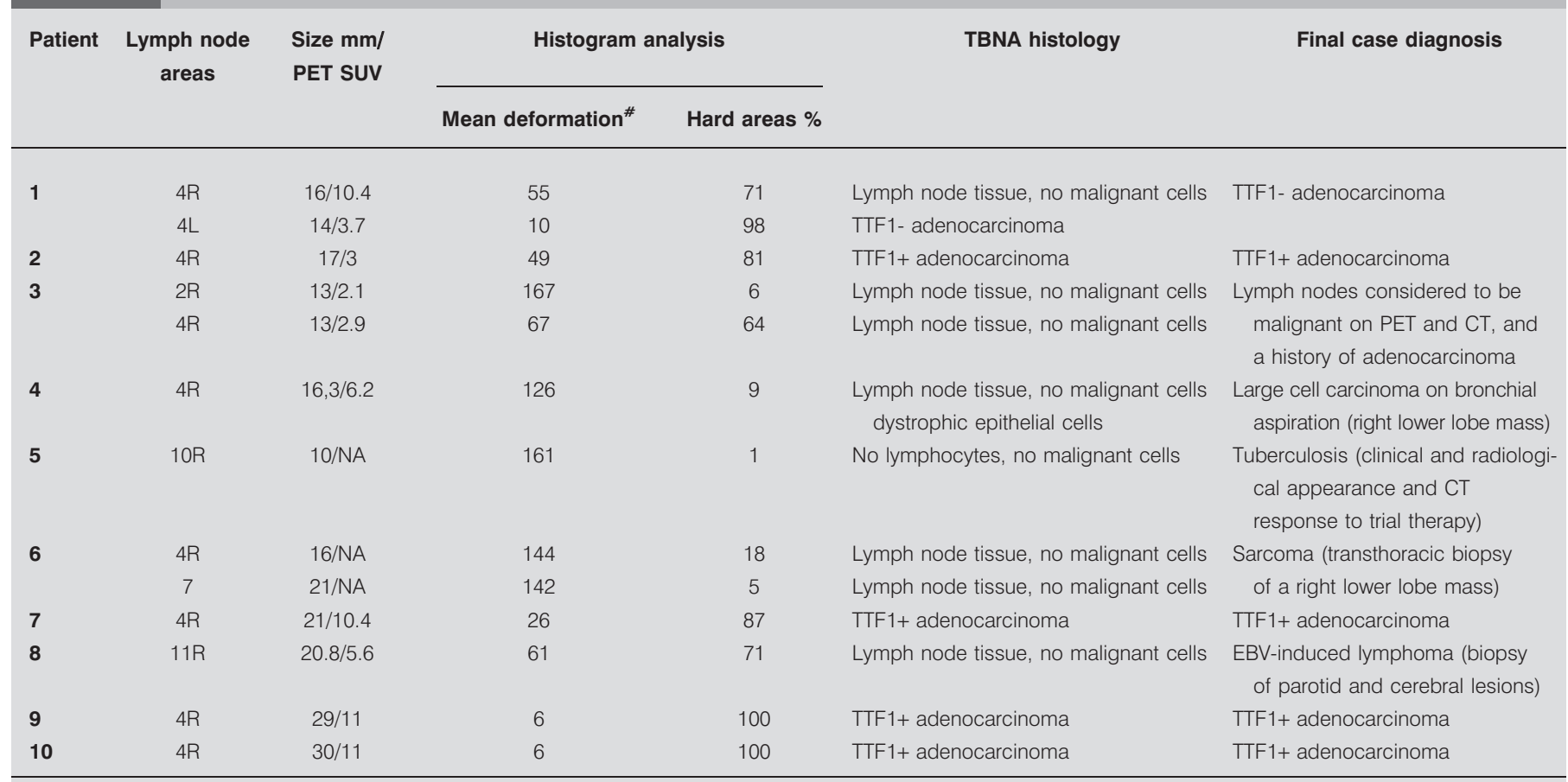

PET: positron emission tomography; SUV: standardised uptake value; TBNA: transbronchial needle aspiration; TTF: thyroid transcription factor; CT: computed tomography; EBV: Epstein-Barr virus; NA: data not available. ${ }^{\#}$ : from 0 to 255. 
studies, the performances of endobronchial elastography will have to be assessed against the results of mediastinoscopy as the gold standard approach.

From this experience, we conclude that transbronchial biomechanical analysis can be performed during bronchial endoscopic ultrasound. Tracheobronchial cartilage does not appear to interfere with collection of this type of information. The preliminary results suggest that elastography could possibly improve the diagnostic yield of bronchial endoscopic ultrasound, as has already been demonstrated in gastrointestinal endoscopy. This is all the more important as endobronchial ultrasonography has a low negative predictive value regarding the neoplastic involvement of mediastinal lymph nodes (see [11] for an example). We propose that our preliminary results justify the setting-up of large-scale studies to precisely describe the operating characteristics of bronchial endoscopic ultrasound elastography. It would also be interesting to determine whether elastography could improve guidance of TBNA in heterogeneous lymph node masses or limit the number of TBNAs performed, by primarily targeting the lymph nodes most likely to be malignant.

\section{Valery Trosini-Désert*,\#, Florence Jeny*, Laurent Taillade Stephane Vignot ${ }^{\oplus}$, Hedi Zribi*, Frédérique Capron ${ }^{+}$and Thomas Similowski* \\ *Assistance Publique - Hôpitaux de Paris, Groupe Hospitalier Pitié-Salpêtrière Charles Foix, Dept of Respiratory and Critical Care Medicine, "Assistance Publique - Hôpitaux de Paris, Groupe Hospitalier Pitié-Salpêtrière Charles Foix, Dept of Medical Oncology, ${ }^{+}$Assistance Publique - Hôpitaux de Paris, Groupe Hospitalier Pitié-Salpêtrière Charles Foix, Dept of Pathology, and "Groupe d'Endoscopie de Langue Française, Société de Pneumologie de Langue Française, Paris, France.}

Correspondence: V. Trosini-Désert, Service de Pneumologie et de Réanimation Médicale, Groupe Hospitalier Pitié-Salpêtrière Charles Foix - Division Montyon, 47-83 Bd de l'Hôpital, 75651 Paris Cedex 13, France. E-mail: valery.trosini-desert@psl.aphp.fr

Support Statement: The "Association Pour la Vie-Espoir Contre le Cancer" provided financial support in the acquisition of bronchial endoscopic ultrasound equipment by the Groupe Hospitalier Pitié-Salpêtrière Charles Foix Dept of Respiratory and Critical Care Medicine (Paris, France).
Acknowledgements: The authors thank D. Khayat (Assistance Publique - Hôpitaux de Paris, Groupe Hospitalier PitiéSalpêtrière Charles Foix, Dept of Medical Oncology, Paris, France) and the "Association Pour la Vie - Espoir Contre le Cancer" (Paris) for their assistance in the acquisition of bronchial endoscopic ultrasound equipment by the Groupe Hospitalier Pitié-Salpêtrière Charles Foix, Dept of Respiratory and Critical Care Medicine.

\section{REFERENCES}

1 Ophir J, Cespedes I, Ponnekanti H, et al. Elastography: a quantitative method for imaging the elasticity of biological tissues. Ultrason Imaging 1991; 13: 111-134.

2 Barr RG, Destounis S, Lackey LB 2nd, et al. Evaluation of breast lesions using sonographic elasticity imaging: a multicenter trial. J Ultrasound Med 2012; 31: 281-287.

3 Shuzhen C. Comparison analysis between conventional ultrasonography and ultrasound elastography of thyroid nodules. Eur J Radiol 2012; 81: 1806-1811.

4 Brock M, von Bodman C, Palisaar RJ, et al. The impact of real-time elastography guiding a systematic prostate biopsy to improve cancer detection rate: a prospective study of 353 patients. J Urol 2012; 187: 2039-2043.

5 Baldewsing RA, Schaar JA, Mastik F, et al. Local elasticity imaging of vulnerable atherosclerotic coronary plaques. Adv Cardiol 2007; 44: 35-61.

6 Cardoso AC, Carvalho-Filho RJ, Stern C, et al. Direct comparison of diagnostic performance of transient elastography in patients with chronic hepatitis B and chronic hepatitis C. Liver Int 2012; 32: 612-621.

7 Iglesias-Garcia J, Larino-Noia J, Abdulkader I, et al. Quantitative endoscopic ultrasound elastography: an accurate method for the differentiation of solid pancreatic masses. Gastroenterology 2010; 139: $1172-1180$

8 Puli SR, Reddy JB, Bechtold ML, et al. Accuracy of endoscopic ultrasound to diagnose nodal invasion by rectal cancers: a metaanalysis and systematic review. Ann Surg Oncol 2009; 16: 1255-1265.

$9 \mathrm{Xu} \mathrm{W}$, Shi J, Zeng X, et al. EUS elastography for the differentiation of benign and malignant lymph nodes: a meta-analysis. Gastrointest Endosc 2011; 74: 1001-1009.

10 Giovannini M, Botelberge T, Bories E, et al. Endoscopic ultrasound elastography for evaluation of lymph nodes and pancreatic masses: a multicenter study. World J Gastroenterol 2009; 15: 1587-1593.

11 Bauwens O, Dusart M, Pierard P, et al. Endobronchial ultrasound and value of PET for prediction of pathological results of mediastinal hot spots in lung cancer patients. Lung Cancer 2008; 61: 356-361.

\section{Fludarabine in paediatric steroid-refractory inflammatory lung injury after stem cell transplantation}

\section{To the Editor:}

In children, the incidence of pulmonary complications after haematopoietic stem cell transplantation (HSCT) varies from
$10 \%$ to $20 \%$. Noninfectious causes, such as idiopathic pneumonia syndrome (IPS), bronchiolitis obliterans syndrome (BOS) and bronchiolitis obliterans organising pneumonia (BOOP), are considered nonspecific inflammatory injuries to the lung [1, 2]. 